\title{
(6) OPEN ACCESS \\ A randomised controlled trial of azithromycin therapy in bronchiolitis obliterans syndrome (BOS) post lung transplantation
}

\author{
Paul A Corris, ${ }^{1,2}$ Victoria A Ryan, ${ }^{3}$ Therese Small, ${ }^{1}$ James Lordan, ${ }^{1}$ Andrew J Fisher, ${ }^{1,2}$ \\ Gerard Meachery, ${ }^{1}$ Gail Johnson, ${ }^{1}$ Chris Ward ${ }^{2}$
}

- Additional material is published online only. To view this file please visit the journal online (http://dx.doi.org/10. 1136/thoraxjnl-2014-205998)

${ }^{1}$ Institute of Transplantation, Freeman Hospital, Newcastle upon Tyne, UK Institute of Cellular Medicine, Newcastle upon Tyne, UK ${ }^{3}$ Institute of Health and Society Newcastle University, Newcastle upon Tyne, UK

\section{Correspondence to} Professor Paul A Corris, Institute of Cellular Medicine, Newcastle University 4th Floor, William Leech Building, Medical School, Framlington Place, Newcastle upon Tyne NE2 4HH, UK;

Paul.corris@nd.ac.uk

Received 4 July 2014 Revised 19 November 2014 Accepted 23 January 2015 Published Online First 24 February 2015

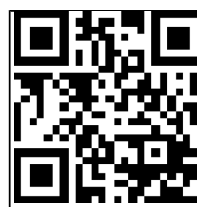

Open Access Scan to access mo free content

\section{SLinked}

- http://dx.doi.org/10.1136/ thoraxjnl-2015-207080

\section{CrossMark}

To cite: Corris PA, Ryan VA, Small T, et al. Thorax 2015:70:442-450

\section{ABSTRACT}

Background We conducted a placebo-controlled trial of azithromycin therapy in bronchiolitis obliterans syndrome (BOS) post lung transplantation.

Methods We compared azithromycin (250 mg alternate days, 12 weeks) with placebo. Primary outcome was $\mathrm{FEV}_{1}$ change at 12 weeks.

Results 48 patients were randomised; (25 azithromycin, 23 placebo). It was established, post randomisation that two did not have BOS. 46 patients were analysed as intention to treat (ITT) with 33 'Completers'. ITT analysis included placebo patients treated with open-label azithromycin after study withdrawal.

Outcome The ITT analysis ( $n=46,177$ observations) estimated mean difference in $\mathrm{FEV}_{1}$ between treatments (azithromycin minus placebo) was $0.035 \mathrm{~L}$, with a $95 \%$ $\mathrm{Cl}$ of $-0.112 \mathrm{~L}$ to $0.182 \mathrm{~L}(\mathrm{p}=0.6)$. Five withdrawals, who were identified at the end of the study as having been randomised to placebo (four with rapid loss in $\mathrm{FEV}_{1}$, one withdrawn consent) had received rescue openlabel azithromycin, with improvement in subsequent $\mathrm{FEV}_{1}$ at 12 weeks. Study Completers showed an estimated mean difference in $\mathrm{FEV}_{1}$ between treatment groups (azithromycin minus placebo) of $0.278 \mathrm{~L}$, with 95\% Cl for the mean difference: $0.170 \mathrm{~L}$ to $0.386 \mathrm{~L}$ $(p=<0.001)$. Nine of 23 ITT patients in the azithromycin group had $\geq 10 \%$ gain in $\mathrm{FEV}_{1}$ from baseline. No patients in the placebo group had $\geq 10 \%$ gain in $\mathrm{FEV}_{1}$ from baseline while on placebo $(p=0.002)$. Seven serious adverse events, three azithromycin, four in the placebo group, were deemed unrelated to study medication. Conclusions Azithromycin therapy improves $\mathrm{FEV}_{1}$ in patients with BOS and appears superior to placebo. This study strengthens evidence for clinical practice of initiating azithromycin therapy in BOS.

Trial registration number EU-CTR, 2006-00048536/GB.

\section{INTRODUCTION}

Lung transplantation can be the only life-sustaining intervention for end-stage lung disease. ${ }^{1}$ Good functional outcomes have been shown, with improved quality of life. ${ }^{2}$ Long-term survival remains limited by the development of the bronchiolitis obliterans syndrome (BOS) however. ${ }^{3}$

The histological lesion of BOS is obliterative bronchiolitis. This is characterised by epithelial alloimmune and non-alloimmune injury. ${ }^{4} 5$

\section{Key messages}

What is the key question?

- A number of international centres have reported a clinically significant response to azithromycin therapy in open studies of lung transplant recipients with bronchiolitis obliterans syndrome (BOS) but there are also negative studies, where azithromycin therapy was not associated with gain in lung function.

What is the bottom line?

- With no randomised placebo controlled studies performed or published there was a clear unmet need, met by this trial, which showed on average that azithromycin was superior to placebo treatment in our study population.

Why read on?

- This study outlines strengthened evidence for the clinical practice of initiating azithromycin therapy for patients who develop BOS post lung transplantation.

Deterioration in allograft function is characterised by the development of progressive, small airway narrowing, fixed airflow limitation, progressive dyspnoea and premature death. ${ }^{6}$ International data shows in excess of $50 \%$ of patients surviving to 5 years after transplantation develop BOS, ${ }^{6}$ limiting 10 -year survival to around $30 \%$. $^{7}$

Therapeutic approaches have ranged from switching immunosuppression ${ }^{6}$ through to initiating cytolytic therapy. Such approaches, including the use of total lymphoid irradiation ${ }^{8}$ have, at best, reduced the rate of decline in graft function in BOS, with significant iatrogenic potential. ${ }^{8}$

In contrast, retrospective studies of macrolides, in particular low dose azithromycin, have indicated that up to $30 \%$ patients with BOS may gain lung function. A number of international centres have reported a clinical response to azithromycin therapy in around a third of patients, ${ }^{9-13}$ with better life expectancy. ${ }^{13}$ There have been negative studies, however, with no gain in lung function. ${ }^{14-16}$ The need for randomised controlled trial data has been highlighted, ${ }^{17}$ but these have not been performed.

We tested the hypothesis that azithromycin therapy is superior to placebo in patients with BOS 
in a randomised double blind placebo controlled study. Some results have been presented as abstracts. ${ }^{18} 19$

\section{METHODS}

\section{Study design}

\section{Randomisation and masking}

This was a single-centre randomised double-blind placebocontrolled parallel group study comparing azithromycin ( $250 \mathrm{mg}$ on alternate days) with placebo over 12 weeks in lung transplant recipients with BOS, with study drug taken in addition to existing medication. Patients were randomly assigned to a treatment arm in a 1:1 ratio using random permuted blocks within strata. Study medication was provided by Bilcare, (Bilcare GCS Europe, Powys, UK) a commercial clinical trial supplier, independent of the manufacturers of azithromycin.

\section{Patient population}

Patients were recruited between November 2006 and December 2010 from the Freeman Hospital.

\section{Withdrawal of patients from study}

Patients who had a rapid and severe deterioration in lung function were withdrawn from the study (for patient details see online supplementary appendix 2). This was defined a priori as a sustained $500 \mathrm{~mL}$ fall in $\mathrm{FEV}_{1}$ from baseline, before the full 12-week course of study treatment, thought to be due to BOS. Patients could also be withdrawn based on the clinical judgement of the responsible clinician. Following withdrawal patients were treated according to the usual centre and international practice, which included the use of open-label azithromycin. The randomised treatment allocation of withdrawn patients remained concealed.

\section{Assessments}

Spirometry

$\mathrm{FEV}_{1}$ and FVC were measured at baseline, week 4, week 8 and week 12 in the Freeman Hospital.

\section{Bronchoscopy, bronchoalveolar lavage and transbronchial biopsy}

Patients underwent bronchoscopy at baseline (prerandomisation) and at final visit (week 12) as previously described. ${ }^{20}$ Transbronchial biopsies were taken at each allograft bronchoscopy, fixed in $10 \%$ formalin, embedded in paraffin, and then stained with haematoxylin and eosin (H\&E) to assess acute vascular and airway inflammation according to standard ISHLT criteria by a pathologist. ${ }^{21}$

\section{Outcome measures}

The primary outcome measure was change in $\mathrm{FEV}_{1}$ from baseline to 12 weeks. Secondary outcome measures reported here are change in FVC from baseline and change in bronchoalveolar lavage (BAL) neutrophils.

\section{Study oversight}

Newcastle University Clinical Trials Unit monitored the study. An independent data monitoring committee was established to assess accumulating recruitment, safety and efficacy data and to oversee the trial conduct (Statistician (chair) and two consultant respiratory physicians).

\section{Sample size}

Estimates of SDs of differences in $\mathrm{FEV}_{1}$ from baseline to 12 weeks in patients with lung transplant with BOS were based on the Freeman Hospital data. ${ }^{41}$ A sample size of 64 patients, 32 patients per randomised group, allowed for $10 \%$ data attrition. A recruitment period of 30 months was estimated to be adequate to recruit 64 patients.

\section{Statistical methods}

The mean difference in $\mathrm{FEV}_{1}$ between treatment groups was estimated using a multilevel random effects model assuming a normal error structure within and between patients. ${ }^{22}$ The models were fitted in MLwiN software (V.2.28). ${ }^{23}$ Random effects models allow appropriate estimation of the treatment effect (and associated SE) taking into account varying numbers of measurements within patients and varying time between measurements. Models were also adjusted for baseline $\mathrm{FEV}_{1}$ and the two randomisation stratification variables. ${ }^{24}$ Model assumptions were checked and analyses omitting possible outliers or influential observations were performed. The secondary outcome measure, FVC, was analysed in the same way.

BAL neutrophil counts and their change from baseline to 12 weeks were summarised and presented as median and IQR.

The intention-to-treat population (ITT) was defined as all randomised patients with BOS who received at least one dose of study drug. The per protocol population was defined as all randomised patients with BOS who followed the protocol and completed 12 weeks of study drug. Completers included all randomised patients with BOS who completed 12 weeks of study drug.

An 'as treated' analysis was also performed; this was a 'post hoc' analysis which had not been described in the statistical analysis plan and as such should be interpreted cautiously. Patients who withdrew or were withdrawn from study drug were treated with open-label azithromycin. The 'as treated' analysis provided an estimate of the treatment effect allowing for treatment to change over time. In this way a patient's measurement contributed to the treatment effect based on the treatment they were receiving at the time the measurement was taken and not the treatment as randomised (for extended details see online supplementary appendix 1$)$.

\section{RESULTS}

\section{Study patients}

The CONSORT flow chart ${ }^{25}$ is presented in figure 1. Patient withdrawals are detailed in the online supplementary appendix 2 (for extended details see online supplementary appendix 1 ).

All 46 patients in the ITT analysis set had baseline and final visit $\mathrm{FEV}_{1}$ measured. Across all visits there were $177 \mathrm{FEV}_{1}$ measurements: 2 patients had $5 \mathrm{FEV}_{1}$ measurements, 36 patients had 4, 7 patients had 3 , and 1 patient had 2 measurements.

There were 33 patients in the Completers analysis set (16 azithromycin, 17 placebo). For the Completers analysis there were $124 \mathrm{FEV}_{1}$ measures; 26 patients with 4 measurements, 6 patients with 3 and 1 patient with 2 measurements.

The 'as treated' analysis used data on all 46 ITT patients and all their $177 \mathrm{FEV}_{1}$ measurements. For the five placebo patients who were withdrawn and placed on open label azithromycin, their measurements post withdrawal (nine measurements in total across the five patients) contributed to the overall azithromycin treatment mean and not the placebo treatment mean.

Baseline characteristics of the study patients, for the ITT and Completer analysis sets, are summarised in table 1. All patients initially received standard immunosuppressant comprising ciclosporin, prednisolone and azathioprine. Patients with more than one episode of vascular rejection requiring augmented steroids in the 1st year post transplant and women with problematic hirsute were switched to tacrolimus treatment. In the ITT population 38 of 46 had switched to tacrolimus by enrolment; 18 in the azithromycin group and 20 in the placebo group. All 


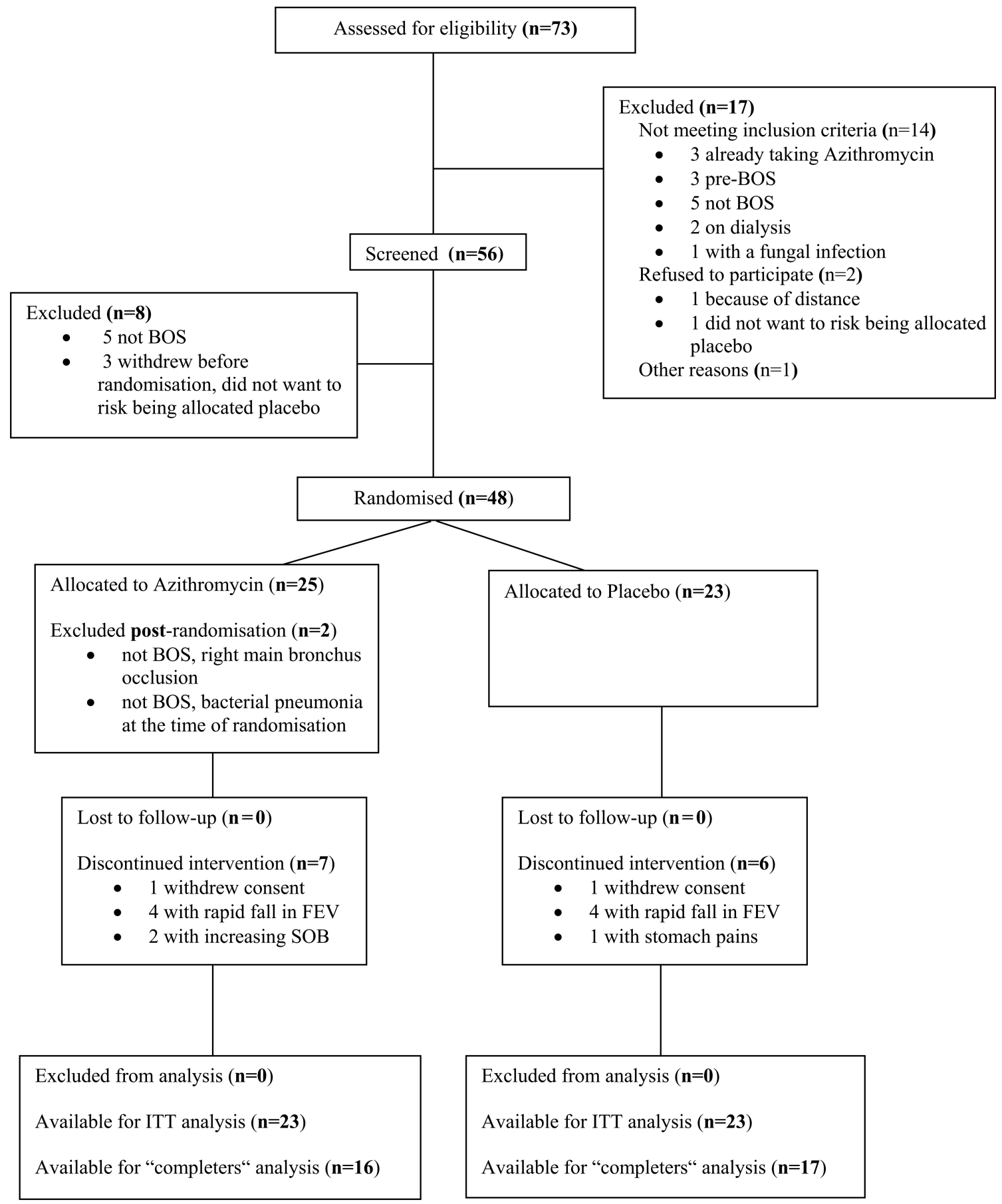

Figure 1 CONSORT flow chart ${ }^{25}$ summarising the progress of patients through the trial. BOS, bronchiolitis obliterans syndrome; ITT, intention-to-treat.

switches were in the 1st year post transplant, well before study enrolment. All patients received proton pump inhibitors and statin therapy, throughout the study. There were no relevant differences in background immunosuppressant or other therapies between the two groups. Results of lavage microbiology did not lead to any change in baseline therapy and no patient enrolled was regarded as having a new infection.

\section{Analysis of $\mathrm{FEV}_{1}$ data}

Figure $2 \mathrm{~A}$ summarises $\mathrm{FEV}_{1}$ measurements as a two-panel spaghetti plot of $\mathrm{FEV}_{1}$ over time in the study. Figure 2B summarises $\mathrm{FEV}_{1}$ measurements in patients who were randomised to the placebo arm and withdrew or were withdrawn and then received open-label azithromycin. Results are summarised in table 2. For the ITT analysis ( $n=46,177$ observations), the estimated mean difference in $\mathrm{FEV}_{1}$ between treatment groups (azithromycin minus placebo) was $0.035 \mathrm{~L}$, (on average higher in the azithromycin group) with a $95 \%$ CI for the mean difference of $-0.112 \mathrm{~L}$ to $0.182 \mathrm{~L}(\mathrm{p}=0.6)$. Nine out of $23(39 \%)$ ITT patients in the azithromycin group had $\geq 10 \%$ gain in $\mathrm{FEV}_{1}$ from baseline. No patients in the placebo arm had $\geq 10 \%$ gain in $\mathrm{FEV}_{1}$ from baseline while on placebo ( $\mathrm{p}<0.002$, Fisher's exact test).

For the 'as treated' analysis ( $n=46,177$ observations) the estimated mean difference in $\mathrm{FEV}_{1}$ between treatment groups 
Table 1 Baseline characteristics for the intention-to-treat (ITT, $n=46)$ and Completer (Comp, n=33) populations, by treatment allocation group

\begin{tabular}{|c|c|c|c|c|}
\hline Baseline characteristic & ITT azithromycin n=23 & ITT placebo $n=23$ & Comp azithromycin $n=16$ & Comp placebo $n=17$ \\
\hline \multicolumn{5}{|l|}{ Sex } \\
\hline Female & $12(52 \%)$ & $8(35 \%)$ & $9(56 \%)$ & $7(41 \%)$ \\
\hline Male & $11(48 \%)$ & $15(65 \%)$ & $7(44 \%)$ & $10(59 \%)$ \\
\hline Age (years), median (IQR) & $51.0(35-56)$ & $51.0(44-59)$ & $53.5(47.0-57.5)$ & $54.0(45.0-62.0)$ \\
\hline \multicolumn{5}{|l|}{ Pretransplant (Tx) disease } \\
\hline Cystic fibrosis & $7(30 \%)$ & $6(26 \%)$ & $2(13 \%)$ & $4(24 \%)$ \\
\hline Emphysema & $11(48 \%)$ & $8(35 \%)$ & $9(56 \%)$ & $6(35 \%)$ \\
\hline Fibrosing alveolitis & $2(9 \%)$ & $4(17 \%)$ & $2(13 \%)$ & $3(18 \%)$ \\
\hline Other* & $3(13 \%)$ & $5(22 \%)$ & $3(19 \%)$ & $4(24 \%)$ \\
\hline \multicolumn{5}{|l|}{ Tx procedure } \\
\hline Double lung & $14(61 \%)$ & $13(57 \%)$ & $7(44 \%)$ & $9(53 \%)$ \\
\hline Single lung & $9(39 \%)$ & $9(39 \%)$ & $9(56 \%)$ & $7(41 \%)$ \\
\hline Heart lung & 0 & $1(4 \%)$ & 0 & $1(6 \%)$ \\
\hline \multicolumn{5}{|l|}{ Years between $\mathrm{Tx}$ and BOS } \\
\hline Median (IQR) & $3.7(1.3-7.4)$ & $2.2(1.3-5.0) \dagger$ & $4.2(2.6-7.8)$ & $2.0(1.3-4.0)$ \\
\hline \multicolumn{5}{|l|}{ BOS stage } \\
\hline 1 & $13(57 \%)$ & $17(74 \%)$ & $10(63 \%)$ & $13(77 \%)$ \\
\hline 2 & $8(35 \%)$ & $4(17 \%)$ & $6(38 \%)$ & $3(18 \%)$ \\
\hline 3 & $2(9 \%)$ & $2(9 \%)$ & 0 & $1(6 \%)$ \\
\hline $\mathrm{FEV}_{1}$ (litres), median (IQR) & $1.5(1.2-2.4)$ & $1.7(1.5-2.5)$ & $1.6(1.2-2.4)$ & $1.7(1.5-2.2)$ \\
\hline FVC (L), median (IQR) & $3.0(2.3-3.6)$ & $2.9(2.2-3.6)$ & $2.7(2.1-3.6)$ & $2.9(2.1-3.5)$ \\
\hline \multicolumn{5}{|l|}{ TBB A and B scores $\ddagger$} \\
\hline Missing & $2(9 \%)$ & $4(17 \%)$ & $2(13 \%)$ & $3(18 \%)$ \\
\hline Ax & $5(22 \%)$ & $8(35 \%)$ & $3(19 \%)$ & $7(41 \%)$ \\
\hline A0 & $12(52 \%)$ & $7(30 \%)$ & $7(44 \%)$ & $4(24 \%)$ \\
\hline $\mathrm{A} 1$ & $4(17 \%)$ & $3(13 \%)$ & $4(25 \%)$ & $2(12 \%)$ \\
\hline $\mathrm{A} 2$ & 0 & $1(4 \%)$ & 0 & $1(6 \%)$ \\
\hline Missing & $2(9 \%)$ & $4(17 \%)$ & $2(13 \%)$ & $3(18 \%)$ \\
\hline $\mathrm{Bx}$ & $5(22 \%)$ & $7(30 \%)$ & $5(31 \%)$ & $4(24 \%)$ \\
\hline BO & $5(22 \%)$ & $6(26 \%)$ & $2(13 \%)$ & $5(29 \%)$ \\
\hline B1R & $9(39 \%)$ & $6(26 \%)$ & $7(44 \%)$ & $5(29 \%)$ \\
\hline$B 2 R$ & $2(9 \%)$ & 0 & 0 & 0 \\
\hline \multicolumn{5}{|l|}{ BAL microbiology } \\
\hline Missing & $2(9 \%)$ & $2(9 \%)$ & $2(13 \%)$ & $1(6 \%)$ \\
\hline NPI & $11(48 \%)$ & $14(61 \%)$ & $8(50 \%)$ & $11(65 \%)$ \\
\hline 'Any' organism & $10(43 \%)$ & $7(30 \%)$ & $6(38 \%)$ & $5(29 \%)$ \\
\hline 'Any' includes: Pa & 5 & 4 & 3 & 3 \\
\hline Asp Fum & 2 & 0 & 1 & 0 \\
\hline $\mathrm{Ca}$ & 4 & 2 & 2 & 1 \\
\hline Other & 2 & 1 & 1 & 1 \\
\hline
\end{tabular}

*Other Pre Tx disease: Obliterative Bronchiolitis, Sarcoid, Congenital heart disease, Histiocytosis X, Silicosis.

tOne patient randomised to the placebo arm $>10$ years post transplant (at 11.9 years).

\#ISHLT grades (ref 20) BAL Microbiology=Clinical microbiology. Other=Proteus mirabilis, Stenotrophomonas Maltophilia, Ralstonia Picketti, Candida species. Percentages for patients growing individual organisms are not given since some patients grew more than one organism.

Asp Fum, Aspergillus fumigatus; BAL, bronchoalveolar lavage; BOS, bronchiolitis obliterans syndrome; Ca, Candida albicans; NPI, no pathogens identified; Pa, Pseudomanas aeruginosa; TBB, transbronchial biopsy.

(azithromycin minus placebo) was $0.306 \mathrm{~L}$, with $95 \%$ CI for the mean difference: $0.181 \mathrm{~L}$ to $0.431 \mathrm{~L}(\mathrm{p}=<0.001)$.

For study Completers the estimated mean difference in $\mathrm{FEV}_{1}$ between treatment groups (azithromycin minus placebo) was $0.278 \mathrm{~mL}$, with $95 \% \mathrm{CI}$ for the mean difference: $0.170 \mathrm{~L}$ to $0.386 \mathrm{~L}(\mathrm{p}=<0.001)$.

\section{Analysis of FVC data}

The results are summarised in table 3 . For the ITT population, ( $n=46,177$ observations), the estimated mean difference in FVC between treatment groups (azithromycin minus placebo) was $0.099 \mathrm{~L}$, with $95 \% \mathrm{CI}$ for the mean difference: $-0.026 \mathrm{~L}$ to $0.224 \mathrm{~L}(\mathrm{p}=0.1)$.
For the 'as treated' analysis ( $\mathrm{n}=46,177$ observations) the estimated mean difference in FVC between treatment groups (azithromycin minus placebo) was $0.272 \mathrm{~L}$, with $95 \%$ CI for the mean difference: $0.158 \mathrm{~L}$ to $0.386 \mathrm{~L}$ $(\mathrm{p}=<0.001)$.

For study Completers, the estimated mean difference in FVC between treatment arms (azithromycin minus placebo) was $0.248 \mathrm{~L}$, with $95 \% \mathrm{CI}$ for the mean difference: $0.115 \mathrm{~L}$ to $0.381 \mathrm{~L}(\mathrm{p}=<0.001)$.

\section{BAL neutrophil data}

BAL data was not available from all patients due to a clinical decision that the sample was either not possible or prudent 

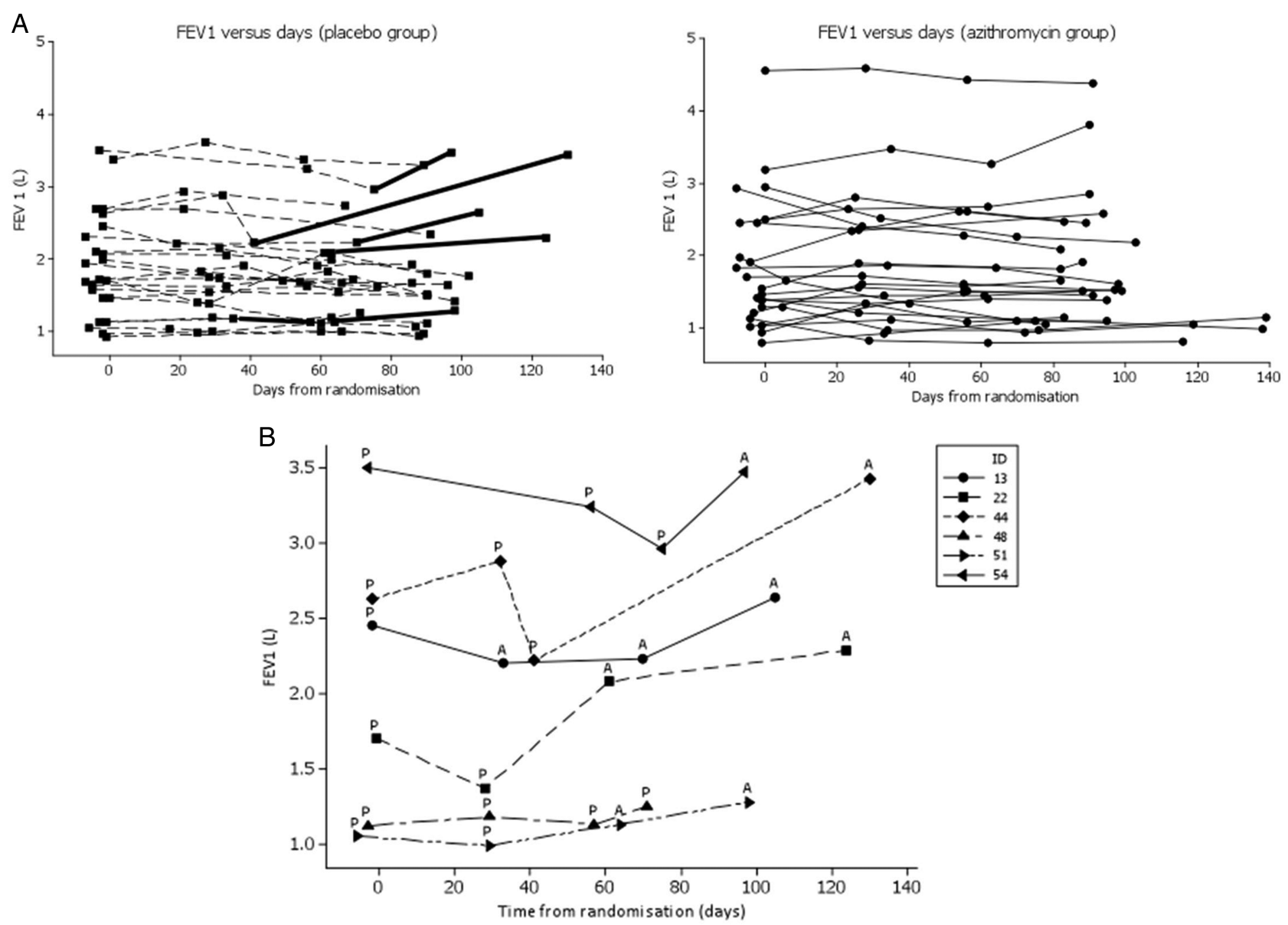

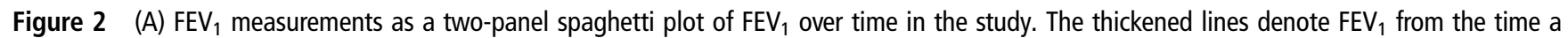
patient withdrew or was withdrawn from study medication. FEV1.0 versus days (placebo group; solid squares) and FEV1.0 versus days (azithromycin group; solid circles). (B) Descriptive plot of $\mathrm{FEV}_{1}$ data for patients treated with placebo who withdrew or were withdrawn from study medication. Symbols, different for each patient (key) denote $\mathrm{FEV}_{1}$ measurements. ID, anonymised patient identifier. ' $P$ ' indicates where a patient was being treated with placebo at the time $\mathrm{FEV}_{1}$ was measured. ' $\mathrm{A}$ ' denotes where a patient was being treated with azithromycin at the time $\mathrm{FEV}_{1}$ was measured, after withdrawal from study. Patient 48 was withdrawn from study medication following stomach pains and did not receive azithromycin. Patient 51 withdrew consent and was treated with open-label azithromycin. The remaining four patients had 'rapid fall' in FEV ${ }_{1}$ and were withdrawn and treated with open label azithromycin.

because of the clinical status of the patients during the bronchoscopy.

At baseline BAL differential data were available for 39/46 $(85 \%)$ of the ITT analysis set. The median per cent neutrophils in BAL was 25.8\% (IQR 3.4-72.0\%).

BAL neutrophil data were available at baseline and final visit for $28 / 46$ patients in the ITT analysis set (13/23 azithromycin, $15 / 23$ placebo) and $25 / 33$ in the Completers analysis set (12/16 azithromycin, $13 / 17$ placebo). Summary statistics for baseline, final and change in BAL neutrophil percentage are given in table 4 . There was no evidence of systematic changes in BAL neutrophil percentage associated with either azithromycin or placebo treatment for either the ITT (figure 3A) or study Completer populations (figure 3B).

\section{Transbronchial biopsy data}

Where paired data were available approximately half of the biopsies were graded as 'Bx' (ungradeable) for the B scores and a third were 'Ax' (ungradeable) for the A scores. Further analysis of the potential effect of azithromycin on biopsy scores was therefore not carried out. The data is summarised in online supplementary appendix 3 .
Safety

Seven adverse events led to hospital admission or the prolonging of existing hospitalisation. They were therefore classified as serious, but not related to study medication. There were no other major safety issues to report with the trial.

\section{DISCUSSION}

To our knowledge this is the first randomised controlled study of azithromycin therapy in BOS. Our trial showed that azithromycin improves $\mathrm{FEV}_{1}$ and $\mathrm{FVC}$ in a significant proportion of patients. As in previous open studies not all patients with BOS responded to azithromycin.

We also noted that in patients treated with placebo who were withdrawn from the study due to rapid fall in lung function, treatment with open label azithromycin was associated with a significant gain in lung function leading to our decision to report our Completers and 'as treated' groups. We recognise that our 'as treated' analysis should be interpreted cautiously, as a post hoc analysis. Overall we conclude that azithromycin appears superior to placebo treatment in our study.

An implication of the present study is that the definition of BOS, which currently includes the presence of irreversible 
Table 2 Mean difference in $\mathrm{FEV}_{1}$ between treatment groups for the intention-to-treat (ITT, $\left.\mathrm{n}=46\right)$, 'as treated' $(\mathrm{n}=46)$ and Completer $(\mathrm{n}=33$ ) populations

\begin{tabular}{|c|c|c|c|}
\hline Outcome FEV 1 (L) & $\begin{array}{l}\text { Mean difference in } \mathrm{FEV}_{1} \\
\text { (azithromycin minus placebo) }\end{array}$ & $\begin{array}{l}95 \% \mathrm{Cl} \text { for population } \\
\text { mean difference }\end{array}$ & p Value \\
\hline & \multicolumn{3}{|c|}{ ITT analysis 46 patients, 177 measurements } \\
\hline \multirow{2}{*}{$\begin{array}{l}\text { Mean difference in } \mathrm{FEV}_{1} \text { between treatment arms, adjusted for baseline } \mathrm{FEV}_{1} \text {, } \\
\text { randomisation stratification variables (disease and transplant) } \\
\text { and time since randomisation }\end{array}$} & 0.035 & -0.112 to 0.182 & 0.6 \\
\hline & \multicolumn{3}{|c|}{ 'As treated' analysis 46 patients, 177 measurements } \\
\hline \multirow{2}{*}{$\begin{array}{l}\text { Mean difference in } \mathrm{FEV}_{1} \text { between treatment arms, adjusted for baseline } \mathrm{FEV}_{1} \text {, } \\
\text { randomisation stratification variables (disease and transplant) and time since randomisation }\end{array}$} & 0.306 & 0.181 to 0.431 & $<0.001$ \\
\hline & \multicolumn{3}{|c|}{ Completers analysis 33 patients, 124 measurements } \\
\hline $\begin{array}{l}\text { Mean difference in } \mathrm{FEV}_{1} \text { between treatment arms, adjusted for baseline } \mathrm{FEV}_{1} \text {, } \\
\text { randomisation stratification variables (disease and transplant) and time since randomisation }\end{array}$ & 0.278 & 0.170 to 0.386 & $<0.001$ \\
\hline
\end{tabular}

airflow obstruction, should be revised or a new term introduced to describe a phenotype of patient who fulfils the definition of BOS, apart from showing a response to azithromycin. This has been suggested prominently by others. ${ }^{5}$

A limitation of our study was randomisation of 48 subjects versus the target of 64 . The incidence of BOS throughout the potential trial population was below that estimated. Consequently, our recruitment rate was on average one per month rather than the estimated rate of two per month. Our recruitment period ran for 48 months rather than the anticipated 30 months. This resulted in having data available from 46 patients for the ITT analysis compared with the target of 58 . This shortfall would be expected to reduce the power of the study. However, the observed SD of the change in $\mathrm{FEV}_{1}$ from baseline was also much smaller than anticipated at design, a factor that would be expected to favour the study power. In line with CONSORT guidelines, ${ }^{25}$ we reported $95 \%$ CIs for the trial, allowing open interpretation of the findings to be considered along with study size.

Our study compliments a previous randomised trial in lung transplantation where azithromycin was used as prophylaxis against developing BOS. This had a primary end point of freedom from BOS and survival 2 years after lung transplantation. $^{26}$ This found that BOS-free survival was better with azithromycin. Patients receiving azithromycin had better $\mathrm{FEV}_{1}$, and lower airway neutrophilia. ${ }^{26}$ When open-label azithromycin was initiated in patients with BOS, this was associated with an improvement of $\mathrm{FEV}_{1}$ in around half the patients treated. ${ }^{26}$
Our study was powered to detect a change in $\mathrm{FEV}_{1} \cdot \mathrm{FEV}_{1}$ is the basis for the internationally recognised classification of BOS and has previously been reported in open studies of azithromycin therapy, ${ }^{9-13}$ and was reported in the sole previous randomised trial of azithromycin for BOS prophylaxis. ${ }^{26} \mathrm{FVC}$ may be sensitive to changes in the calibre of smaller, peripheral airways. $^{27} 28$ Our data indicated that azithromycin was superior to placebo for FVC in the Completer population and 'as treated' analysis.

Our current and previously reported data $^{29}$ therefore suggest that FVC and other tests such as $\mathrm{FEF}_{25-75 \%}$ may be useful end points for intervention studies in BOS. Previous open studies of azithromycin therapy, including work from our own centre have not reported FVC data ${ }^{9-13}$ or focused on physiological measurements which reflect smaller airway function. It would seem reasonable to report such measurements given the recognised small airway contribution to BOS pathophysiology, and that the measurements are readily made.

As a secondary end point we analysed the effects of azithromycin therapy on BAL neutrophils, reflecting our long-standing interest $^{30} 31$ BOS has a neutrophilic pathophysiology and it has been suggested that the clinical heterogeneity of BOS may be clarified by considering a distinct patient subset with neutrophilic reversible allograft dysfunction. ${ }^{5}$ This proposed dichotomy may have important therapeutic implications in predicting patients who might respond to azithromycin. ${ }^{5}$ In a significant number of our patients no BAL data were available. The samples that were available confirmed our previous finding, ${ }^{30}$ and others ${ }^{32} 33$ that BOS is accompanied by an elevated BAL

Table 3 Mean difference in FVC between treatment groups for the intention-to-treat (ITT, $n=46)$, 'as treated' $(n=46)$ and Completer $(n=33)$ populations

\begin{tabular}{|c|c|c|c|}
\hline Outcome FVC (L) & $\begin{array}{l}\text { Mean difference in FVC } \\
\text { (azithromycin minus placebo) }\end{array}$ & $\begin{array}{l}95 \% \mathrm{Cl} \text { for population } \\
\text { mean difference }\end{array}$ & p Value \\
\hline & \multicolumn{2}{|c|}{ ITT analysis 46 patients, 177 measurements } & \\
\hline \multirow{2}{*}{$\begin{array}{l}\text { Mean difference in FVC between treatment arms, adjusted for baseline FVC, randomisation } \\
\text { stratification variables (disease and transplant) and time since randomisation }\end{array}$} & 0.099 & -0.026 to 0.224 & 0.1 \\
\hline & \multicolumn{2}{|c|}{ 'As treated' analysis 46 patients, 177 measurements } & \\
\hline \multirow{2}{*}{$\begin{array}{l}\text { Mean difference in FVC between treatment arms, adjusted for baseline FVC, randomisation } \\
\text { stratification variables (disease and transplant) and time since randomisation }\end{array}$} & 0.272 & 0.158 to 0.386 & $<0.001$ \\
\hline & \multicolumn{2}{|c|}{ Completers analysis 33 patients, 124 measurements } & \\
\hline $\begin{array}{l}\text { Mean difference in FVC between treatment arms, adjusted for baseline FVC, randomisation } \\
\text { stratification variables (disease and transplant) and time since randomisation }\end{array}$ & 0.248 & 0.115 to 0.381 & $<0.001$ \\
\hline
\end{tabular}


Table 4 Per cent neutrophils in bronchoalveolar lavage at baseline and final visit (week 12) for the intention-to-treat (ITT) (n=28/46) and Completer $(n=25 / 33)$ populations, by treatment allocation

\begin{tabular}{llll}
\hline & $\mathbf{n}$ & $\begin{array}{l}\text { Baseline } \\
\text { Median (IQR) }\end{array}$ & $\begin{array}{l}\text { Change from baseline } \\
\text { Median (IQR) }\end{array}$ \\
\hline ITT azithromycin & $13 / 23$ & $16.6(4.2$ to 68.8$)$ & $32.0(10.0$ to 69.5$)$ \\
ITT placebo & $15 / 23$ & $14.8(2.4$ to 56.0$)$ & $19.8(2.0$ to 52.2$)$ \\
Completers azithromycin & $12 / 16$ & $16.1(3.7$ to 61.5$)$ & $31.5(7.5$ to 73.3$)$ \\
Completers placebo & $13 / 17$ & $9.2(2.0$ to 52.5$)$ & 19.4 to 17.7$)$ \\
\hline
\end{tabular}

neutrophil count. However azithromycin therapy was not associated with a change in neutrophils. Our findings are therefore different to the Leuven Centre which has published data indicating that a fall in neutrophilic inflammation occurs in patients with a clinical response to azithromycin. We feel that the limited data available for analysis in our study precludes firm conclusions being drawn. We would therefore recommend that further research is performed to clarify the relationship between BAL neutrophil levels and the clinical effectiveness of azithromycin treatment.

Apart from potential anti-inflammatory benefits, which may include effects on neutrophil numbers and function, other effects of macrolide therapy have been reviewed elsewhere and warrant further study. These include immunomodulatory mechanisms, interference in the formation of infective biofilms and alleviation of extraoesophageal reflux and microaspiration, with promotion of gastric motility. ${ }^{34}$ It is increasingly recognised that reflux and aspiration may be an important injury in lung allografts, ${ }^{35}$ and we would recommend that characterisation of reflux disease is considered in future intervention trials in BOS.

In our study azithromycin treatment was not associated with significant adverse events. There were no deaths or graft losses during the study. It has been suggested that prolonged treatment with azithromycin may have adverse effects which may be of potential relevance in lung transplantation. These include gastrointestinal effects, loss of hearing ${ }^{36}$ and the development of macrolide resistant organisms. ${ }^{34}$ It is also suggested that azithromycin could predispose patients to the development of non-TB
A

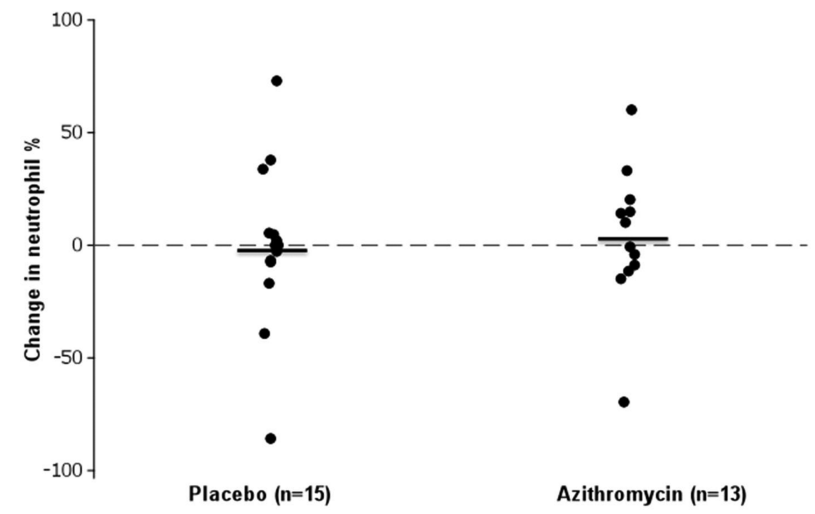

C

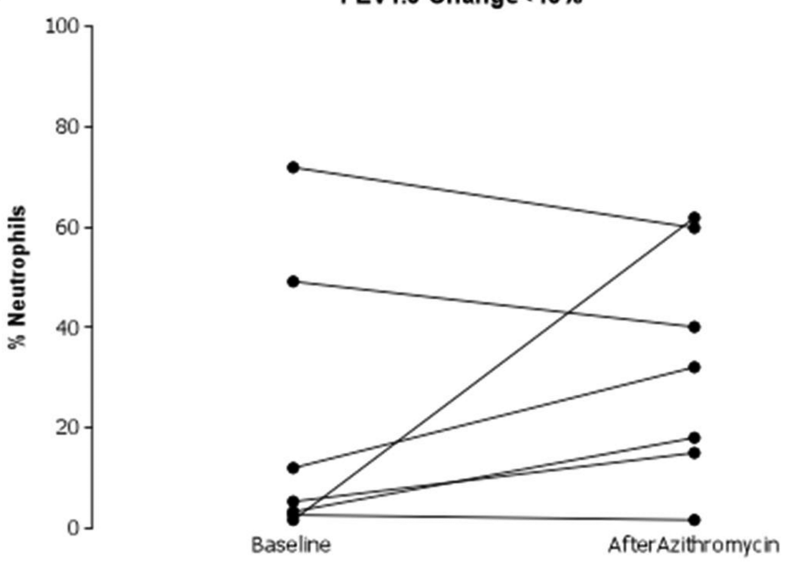

B

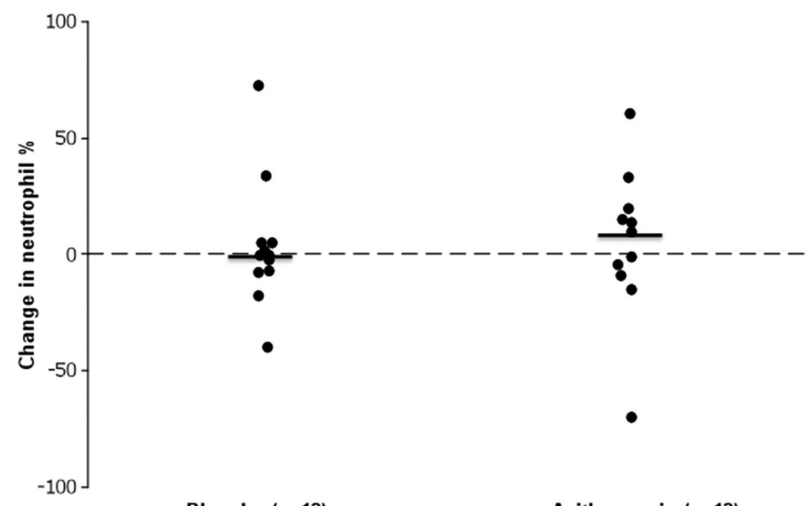

Placebo $(n=13)$

Azithromycin ( $n=12)$

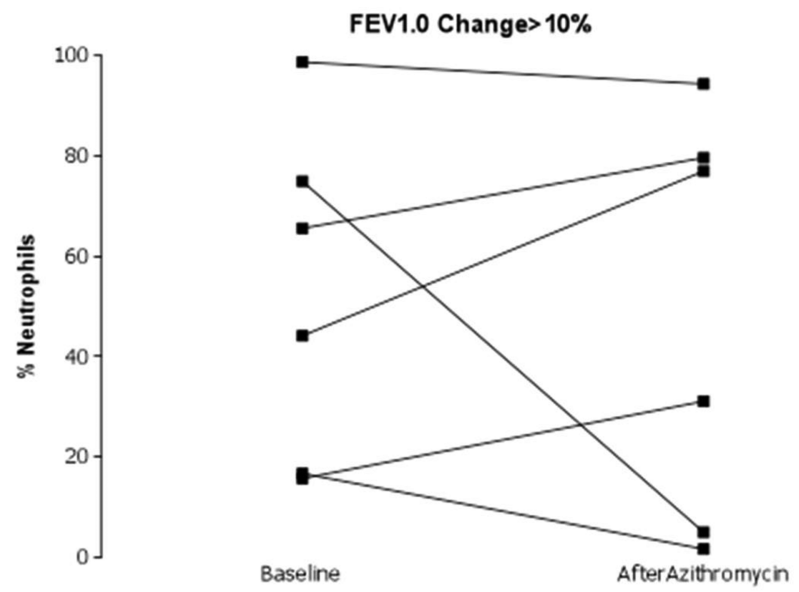

Figure 3 (A) The change in per cent neutrophils in bronchoalveolar lavage (BAL) from baseline to week 12 for the intention-to-treat (ITT) population, by treatment allocation group $(n=28 / 46)$. Median change denoted by horizontal line. (B) The change in per cent neutrophils in $B A L$ from baseline to week 12 for the Completer population, by treatment allocation group ( $n=25 / 33$ ). Median change denoted by horizontal line. (C) The change in per cent neutrophils in baseline to week 12 for patients treated with azithromycin who had $<10 \%$ gain in $\mathrm{FEV}_{1}$ (solid circles) and in patients treated with azithromycin who had a $>10 \%$ gain in $\mathrm{FEV}_{1}$ (solid squares). 
mycobacterial infection. ${ }^{37}$ Debate has also been generated by a study of azithromycin use in a group of patients with preexisting heart disease. Here azithromycin treatment was associated with an increased rate of cardiovascular related mortality. ${ }^{38}$ It has also been shown that the macrolide antibiotics may cause cholestatic hepatitis at an estimated rate of 3.6 per $100000 .^{39}$

While azithromycin treatment in BOS is generally safe, and provides a therapeutic opportunity in a pathophysiology causing significant morbidity and mortality, ${ }^{9} 1012-162640$ it remains a research priority to elucidate which patients benefit from azithromycin, what the optimum timing of treatment is and to provide long-term follow-up data. This might lessen the possibility of iatrogenic consequences of therapy, although these are also the subject of investigation and debate, with the possibility that risks have been overestimated generally, and may not be especially relevant in the specialised setting of lung transplantation. We consider the potential benefits of alternate day low dose azithromycin $250 \mathrm{mg}$ outweigh the potential risks in lung transplantation. Ideally the results of this trial should be replicated.

We conclude that this study provides strengthened evidence for the clinical practice of initiating azithromycin therapy for patients who develop BOS post lung transplantation.

Acknowledgements The authors thank all staff in the Freeman Hospital transplant unit. The authors also thank the volunteer data monitoring and ethics committee for the trial; Professor Martin Bland (Professor of Health Statistics at the University of York), Dr Chris Stenton (Consultant Respiratory Physician, Royal Victoria Infirmary, Newcastle upon Tyne) and Dr Graham Burns (Consultant Respiratory Physician, Royal Victoria Infirmary, Newcastle upon Tyne). This research was only possible due to the courage of the participant lung transplant recipients and their families.

Contributors PAC was clinical science lead for this study. He co-wrote the manuscript; VAR was trial statistician. She analysed the data, wrote the statistical sections and helped to write the manuscript; TS was trial manager and reviewed the manuscript; JL, AJF, GM were clinical co-investigators, who recruited patients and reviewed the manuscript; GJ was lab scientist responsible for all patient BAL sample results and reviewed the manuscript; CW was science lead and principal investigator for this study. He wrote the manuscript drafts.

Funding Funded by Medical Research Council Project grant G0500705 and a British Lung Foundation Trevor Clay Award.

\section{Competing interests None.}

Patient consent Obtained.

Ethics approval Local ethics committee and UK Medicines and Healthcare products Regulatory Agency (MHRA).

Provenance and peer review Not commissioned; externally peer reviewed.

Open Access This is an Open Access article distributed in accordance with the terms of the Creative Commons Attribution (CC BY 4.0) license, which permits others to distribute, remix, adapt and build upon this work, for commercial use, provided the original work is properly cited. See: http://creativecommons.org/ licenses/by/4.0/

\section{REFERENCES}

Arcasoy SM, Kotloff RM. Lung transplantation. N Engl J Med 1999;340:1081-91.

2 Rutherford RM, Fisher AJ, Hilton C, et al. Functional status and quality of life in patients surviving 10 years after lung transplantation. Am J Transplant 2005;5:1099-104.

3 Estenne M, Maurer JR, Boehler A, et al. Bronchiolitis obliterans syndrome 2001: an update of the diagnostic criteria. J Heart Lung Transplant 2002;21:297-310.

4 Diamond JM, Lee JC, Kawut SM, et al. Clinical risk factors for primary graft dysfunction after lung transplantation. Am J Respir Crit Care Med 2013;187:527-34.

5 Vanaudenaerde BM, Meyts I, Vos R, et al. A dichotomy in bronchiolitis obliterans syndrome after lung transplantation revealed by azithromycin therapy. Eur Respir J 2008:32:832-43.

6 Todd JL, Palmer SM. Bronchiolitis obliterans syndrome: the final frontier for lung transplantation. Chest 2011;140:502-8.
7 Christie JD, Edwards LB, Kucheryavaya AY, et al. The registry of the International Society for Heart and Lung Transplantation: twenty-seventh official adult lung and heart-lung transplant report-2010. J Heart Lung Transplant 2010;29: 1104-18.

8 Fisher AJ, Rutherford RM, Bozzino J, et al. The safety and efficacy of total lymphoid irradiation in progressive bronchiolitis obliterans syndrome after lung transplantation. Am J Transplant 2005;5:537-43.

9 Gerhardt SG, McDyer JF, Girgis RE, et al. Maintenance azithromycin therapy for bronchiolitis obliterans syndrome: results of a pilot study. Am J Respir Crit Care Med 2003; 168:121-5

10 Verleden GM, Dupont LJ. Azithromycin therapy for patients with bronchiolitis obliterans syndrome after lung transplantation. Transplantation 2004;77:1465-7.

11 Yates B, Murphy DM, Forrest IA, et al. Azithromycin reverses airflow obstruction in established bronchiolitis obliterans syndrome. Am I Respir Crit Care Med 2005:172:772-5.

12 Gottlieb J, Szangolies J, Koehnlein T, et al. Long-term azithromycin for bronchiolitis obliterans syndrome after lung transplantation. Transplantation 2008;85:36-41.

13 Jain R, Hachem RR, Morrell MR, et al. Azithromycin is associated with increased survival in lung transplant recipients with bronchiolitis obliterans syndrome. I Heart Lung Transplant 2010;29:531-7.

14 Shitrit $\mathrm{D}$, Bendayan $\mathrm{D}$, Gidon $\mathrm{S}$, et al. Long-term azithromycin use for treatment of bronchiolitis obliterans syndrome in lung transplant recipients. J Heart Lung Transplant 2005;24:1440-3.

15 Porhownik NR, Batobara W, Kepron W, et al. Effect of maintenance azithromycin on established bronchiolitis obliterans syndrome in lung transplant patients. Can Respir J 2008;15:199-202.

16 Benden C, Boehler A. Long-term clarithromycin therapy in the management of lung transplant recipients. Transplantation 2009:87:1538-40.

17 Williams TJ, Verleden GM. Azithromycin: a plea for multicenter randomized studies in lung transplantation. Am J Respir Crit Care Med 2005;172:657-9.

18 Corris P, Small T, Ryan V, et al. A Randomised Controlled Trial Of Azithromycin Therapy In Bronchiolitis Obliterans Syndrome (BOS) Post Lung Transplantation. Am J Respir Crit Care Med 2012;315:A5324.

19 Corris PA, Small T, Ryan VA, et al. 173 A randomised controlled trial of azithromycin therapy in Bronchiolitis Obliterans Syndrome (BOS) post lung transplantation. J Heart Lung Transplant 2012;31:S67.

20 Forrest IA, Murphy DM, Ward C, et al. Primary airway epithelial cell culture from lung transplant recipients. Eur Respir J 2005;26:1080-5.

21 Stewart S, Fishbein MC, Snell Gl, et al. Revision of the 1996 working formulation for the standardization of nomenclature in the diagnosis of lung rejection. $J$ Heart Lung Transplant 2007;26:1229-42.

22 Steele F. Multilevel models for longitudinal data. J R Stat Soc Ser A Stat Soc 2008:171:5-19.

23 Rasbash J, Browne W, Healey W, et al. MLwiN Version 2.28. London: Institute of Education, Multilevel Models Project, 2013.

24 Senn S. Change from baseline and analysis of covariance revisited. Stat Med 2006;25:4334-44.

25 Schulz KF, Altman DG, Moher D. CONSORT 2010 statement: updated guidelines for reporting parallel group randomised trials. BMJ 2010;340:c332.

26 Vos R, Vanaudenaerde BM, Verleden SE, et al. A randomised controlled trial of azithromycin to prevent chronic rejection after lung transplantation. Eur Respir $J$ 2011;37:164-72

27 Burns GP. Respiratory medicine. In: Bourke SJ, Burns GP, eds. Respiratory medicine lecture notes. UK: Wiley Blackwell, 2011:6-7.

28 Contoli M, Bousquet J, Fabbri LM, et al. The small airways and distal lung compartment in asthma and COPD: a time for reappraisal. Allergy 2010;65:141-51.

29 Chacon RA, Corris PA, Dark JH, et al. Tests of airway function in detecting and monitoring treatment of obliterative bronchiolitis after lung transplantation. J Heart Lung Transplant 2000;19:263-9.

30 Ward C, Snell Gl, Zheng L, et al. Endobronchial biopsy and bronchoalveolar lavage in stable lung transplant recipients and chronic rejection. Am J Respir Crit Care Med 1998;158:84-91.

31 Kelly C, Ward C, Stenton CS, et al. Number and activity of inflammatory cells in bronchoalveolar lavage fluid in asthma and their relation to airway responsiveness. Thorax 1988:43:684-92.

32 DiGiovine B, Lynch JP III, Martinez FJ, et al. Bronchoalveolar lavage neutrophilia is associated with obliterative bronchiolitis after lung transplantation: role of IL-8. J Immunol 1996;157:4194-202.

33 Verleden GM, Vos R, De Vleeschauwer $\mathrm{Sl}$, et al. Obliterative bronchiolitis following lung transplantation: from old to new concepts? Transpl Int 2009:22:771-9.

34 Murphy DM, Forrest IA, Curran D, et al. Macrolide antibiotics and the airway: antibiotic or non-antibiotic effects? Expert Opin Investig Drugs 2010;19:401-14.

35 Griffin SM, Robertson AG, Bredenoord AJ, et al. Aspiration and allograft injury secondary to gastroesophageal reflux occur in the immediate post-lung transplantation period (prospective clinical trial). Ann Surg 2013;258:705-11; discussion 11-2. 
36 Kanoh S, Rubin BK. Mechanisms of action and clinical application of macrolides as immunomodulatory medications. Clin Microbiol Rev 2010;23: 590-615.

37 Renna M, Schaffner C, Brown K, et al. Azithromycin blocks autophagy and may predispose cystic fibrosis patients to mycobacterial infection. J Clin Invest 2011;121:3554-63.

38 Ray WA, Murray KT, Hall K, et al. Azithromycin and the risk of cardiovascular death. N Engl J Med 2012;366:1881-90.
39 Leitner JM, Graninger W, Thalhammer F. Hepatotoxicity of antibacterials: Pathomechanisms and clinical. Infection 2010:38:3-11.

40 Verleden GM, Vanaudenaerde BM, Dupont LJ, et al. Azithromycin reduces airway neutrophilia and interleukin-8 in patients with bronchiolitis obliterans syndrome. Am J Respir Crit Care Med 2006;174:566-70.

41 Forrest IA. The potential role of matrix metalloproteinases and their inhibitors in obliterative bronchiolitis following lung transplantation. PhD thesis. UK: Newcastle University, 2009. 